\title{
Optical Multi-ring Cascade Cavity Temperature Sensor with Ultra-High Sensitivity
}

Shen San-min ${ }^{1,2}$, Deng Jia-hao ${ }^{1}$ and Yan Shu-bin ${ }^{2^{*}}$

${ }^{1}$ School of Mechatronical Engineering, Beijing Institute of Technology, Beijing 100081, China

${ }^{2}$ Key Laboratory of Instrumentation Science and Dynamic Measurement (Ministry of Education), North University of China, Taiyuan, Shanxi, China

\begin{abstract}
An ultra-small integrated photonic ten-order resonators temperature sensor has been proposed and demonstrated and fabrication using well-developed MEMS technologies incorporates a silicon ring resonator. The temperature variation is measured by monitoring the shift in the ten-order resonant wavelength of the silicon resonator, which was induced by the thermo-optic effect and the thermal expansion effect. The best achieved sensitivity is about $130 \mathrm{pm} /{ }^{\circ} \mathrm{C}$ for a waveguide width of $450 \mathrm{~nm}$, and the radius of the micro ring is about $5 \mu \mathrm{m}$. Meanwhile, the two-channel sensor based on double racetrack rings is also reported.
\end{abstract}

Keywords: Ten-order resonators; Temperature sensor; Thermooptic effect; Thermal expansion effect

\section{Introduction}

Recently silicon photonics technology has received much attention for application in optical communications, temperature sensor, high speed optical modulators, circuit integrated and detectors integrated with silicon waveguides [1-6]. Therefore, high-Q optical waveguide cavity has been widespread concern due to its high integration, small volume mode and high sensitivity. Recently, a lot of articles on the temperature characteristics of the silicon micro-ring resonator or fiber-optic temperature sensors based on Bragg gratings were mainly attempted featuring a high sensitivity and easy fabrication $[7,8]$. But integrate electrical/optical cascade resonators temperature characteristics have not been reported. The high index contrast waveguides are very sensitive to small variations in dimension, refractive index, and especial temperature $[9,10]$. Meanwhile, the ultrasmall dimension of the silicon resonator is expected to help extend the sensing temperature range due to its broad free spectral range (FSR) [11].

In the paper, we fabricated a high performance temperature sensing based on SOI ten-order micro ring resonators. The radius of the micro ring is about $5 \mu \mathrm{m}$, after thermal tuning, we achieved a high-efficiency temperature sensing function in the broadband range. Meanwhile, we fabricated a 2-channel temperature sensing based on a double ring, and obtain some meaningful testing results from these devices.

\section{Design and Fabrication}

As it all know that smaller dimensions are needed to keep waveguides single mode, and smaller gaps between waveguides are needed to allow weakly coupled structures with a limited length. High-index contrast structures are more sensitive to geometrical and refractive index deviations. The devices were fabricated with SOI material, with a top $\mathrm{Si}$ layer of $220 \mathrm{~nm}$ thicknesses, an isolation $\mathrm{SiO}_{2}$ layer of $3 \mu \mathrm{m}$ thickness and a cover $\mathrm{SiO}_{2}$ layer of $2 \mu \mathrm{m}$ thickness. The fabrication procedure was demonstrated as follows: SOI wafer pre-processing, photo resist coating, exposed in PMMA with the $50 \mathrm{kV}$ EBL system, photography development, ICP etching around the Si waveguide core area, photo resist stripping, covering layer $\mathrm{SiO}_{2}$ produced via plasma enhanced chemical vapor deposition (PECVD).

The structure of the device is illustrated in Figure 1. It shows the cross section of the waveguide, with a Si core area of $450 \mathrm{~nm}$ width and $220 \mathrm{~nm}$ height, a $\mathrm{SiO}_{2}$ area of $2 \mu \mathrm{m}$ cladding layer and a $3 \mu \mathrm{m}$ isolation layer. The structure of the filters is illustrated in Figure 2. Figure 2a shows a scanning-electron micrographs (SEM) photograph of the micro ring resonator temperature sensor, including the bus line waveguide, drop line waveguide and a tenth-order micro ring resonator. From Figure $2 \mathrm{a}$, we can figure out that the radius of the micro ring is about $5 \mu \mathrm{m}$ and the gap between the micro ring and the straight waveguide is about $150 \mathrm{~nm}$.

In addition, we fabricated high-order temperature sensor based on a single ring and a racetrack ring. Figure $2 \mathrm{~b}$ shows a two channel temperature sensor based on double racetrack micro-ring. Figure $2 \mathrm{c}$ shows the sidewall of the waveguide about the gap areas.

\section{Results}

The optical measurement setup for the temperature sensor micro ring resonator is shown in Figure 3. In experiments, a tunable laser (1550 $\mathrm{nm}$ band) is used as the optical source to characterize responses of micro-ring resonators. Before the laser is coupled into the bus waveguide through a lensed single mode fiber tip, it will be enlarge through an erbium-doped fiber amplifier. To obtain a

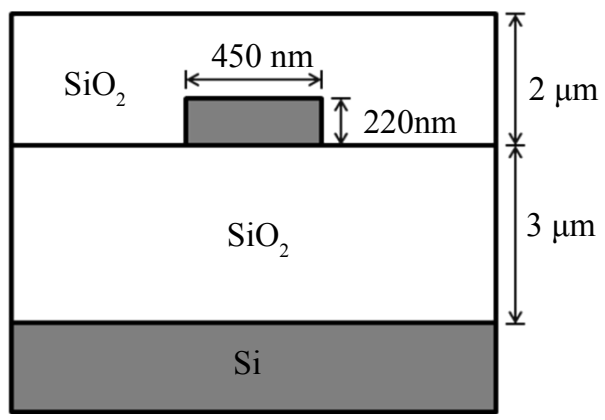

Figure 1: Crosssection of waveguides.

*Corresponding author: Yan Shu-bin, Key Laboratory of Instrumentation Science and Dynamic Measurement (Ministry of Education), North University of China Taiyuan, Shanxi, China, Tel: +86 351392 2018; E-mail: shubin_yan@nuc.edu.cn

Received May 21, 2018; Accepted May 26, 2018; Published June 05, 2018

Citation: San-min S, Jia-hao D, Shu-bin Y (2018) Optical Multi-ring Cascade Cavity Temperature Sensor with Ultra-High Sensitivity. Int J Sens Netw Data Commun 7: 155 doi: 10.4172/2090-4886.1000155

Copyright: $\odot 2018$ San-min S, et al. This is an open-access article distributed under the terms of the Creative Commons Attribution License, which permits unrestricted use, distribution, and reproduction in any medium, provided the original author and source are credited. 
power transmission spectrum, the laser wavelength is swept at a step continuously while the output power of each wavelength is recorded sequentially. The tunable range of laser wavelengths is $1520-1570 \mathrm{~nm}$. The output is collected by another lensed single mode fiber tip and then fed into a photodetector. Finally, the power transmission spectrum can be recorded via an oscilloscope or power meter connected with photodetector. The completed temperature sensor is evaluated by mounting it on a holder placed on a precision stage, whose temperature is adjusted by a built-in thermoelectric cooler and monitored in situ by a k-type thermocouple.

The normalized measured responses of the micro ring temperature sensor shown as Figure 4. It shows three peak responses at the length from $1528 \mathrm{~nm}$ to $1570 \mathrm{~nm}$ and the free spectral range (FSR) reaching $\sim 18 \mathrm{~nm}$, and the bandwidth $\sim 0.14 \mathrm{~nm}$. The corresponding quality factor (Q-factor) was $\sim 11,000$, which is high enough to allow for a high sensing resolution.

The thermo-optic (TO) coefficient of silicon is $\sim 1.86 \times 10^{-4} /{ }^{\circ} \mathrm{C}$. When the temperature varies, the refractive index of the silicon is altered by the TO effect and the response point of the ring is changed by the thermal expansion effect. The overall shift in the resonant wavelength $\Delta \lambda$ is shown as [11,12]:

$$
\Delta \lambda=\Delta \lambda_{L}+\Delta \lambda_{T}=m \alpha \frac{n_{\text {eff }}}{n_{g}} \lambda \Delta T+\frac{\sigma_{T}}{n_{g}} \lambda \Delta T
$$

where $\Delta \lambda \mathrm{L}$ and $\Delta \lambda \mathrm{T}$ are the shift of wavelength because of thermal expansion effect and TO effect respectively; neff is the effective index of

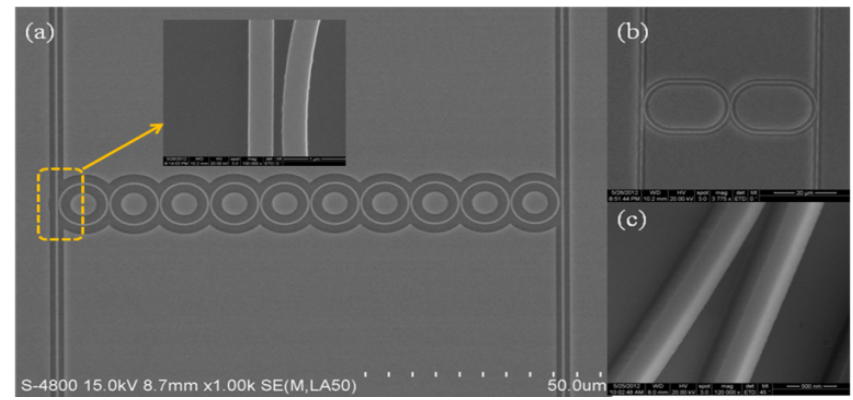

Figure 2: Structures of filters. (a) SEM of tenth-order temperature sensor. (b) SEM of a two-channel temperature sensor based on double racetrack ring. (c) SEM of the sidewall waveguide.

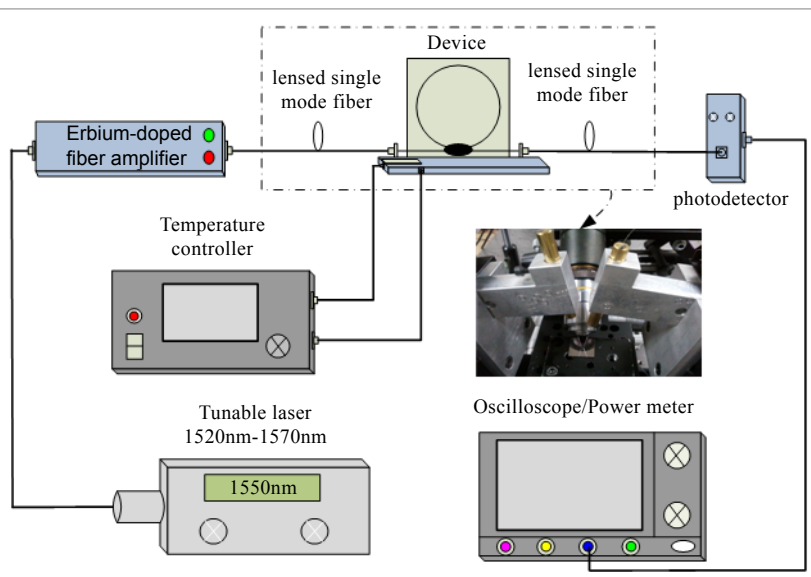

Figure 3: Schematic diagram of the measurement system. the waveguide, $\mathrm{ng}$ is the group index of the waveguide, $\mathrm{m}$ is the number of the resonant, $\alpha$ is the coefficient of thermal expansion (CTE), $\Delta \mathrm{T}$ is the temperature change, and $\sigma \mathrm{T}$ is the rate of change of the effective index with the current temperature $\mathrm{T}$, and it depends on the thermooptic coefficient of the core material and cladding material. $\sigma \mathrm{T}$ is calculated as follows:

$$
\sigma_{T}=\frac{d n_{e f f}}{d T}
$$

Some of the crucial parameters used for the analysis are: the TO coefficients of the $\mathrm{SiO} 2$ is $\sim 1.0 \times 10^{-5} /{ }^{\circ} \mathrm{C}$, and silicon is used substrate and the CTE $\alpha$ is $\sim 2.6 \times 10^{-6} /{ }^{\circ} \mathrm{C}$ while that of the oxide layer is ignored due to the thickness of the silicon substrate is much larger than the oxide layer [11-13]. In this paper, the observed effective index and group index are neff $=\sim 2.85$ and $\mathrm{ng}=\sim 3.89$, therefore, when the sensor wavelength changes 5.2 $\mathrm{nm}$ around $1550 \mathrm{~nm}$, it can be calculated that the temperature variation of the waveguides is about $40^{\circ} \mathrm{C}$ shown as Figure 5 .

From this theoretical result, we can draw this conclusion: when the temperature rises by $1^{\circ} \mathrm{C}$, the resonant wavelength results in 110 $\mathrm{nm}$. The relationship between wavelength and temperatures is shown as Figure 6. Compared with testing result of $130 \mathrm{~nm} /{ }^{\circ} \mathrm{C}$, we can see that the experiment coincides fairly well with theoretical calculation.

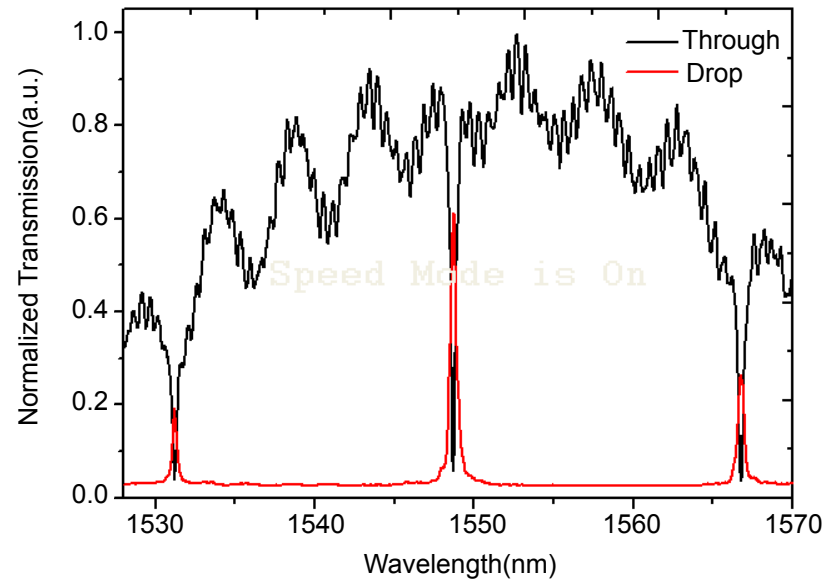

Figure 4: the response of the single micro ring temperature sensor.

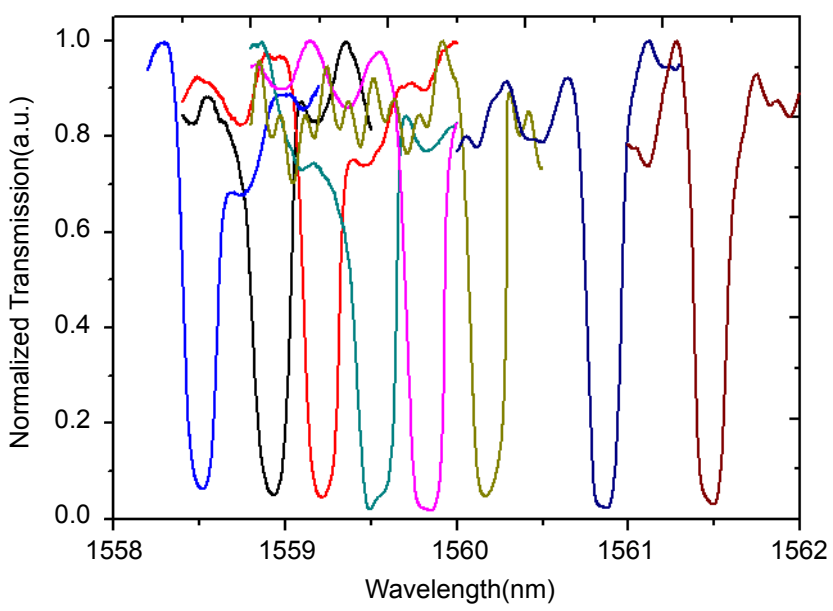

Figure 5: Normalized wavelength responses at different temperatures. 


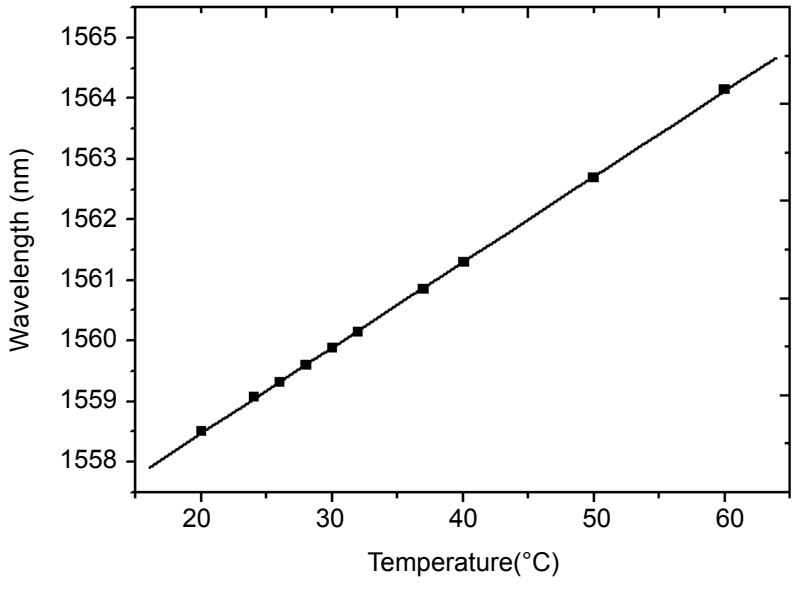

Figure 6: The relationship between wavelength and temperatures

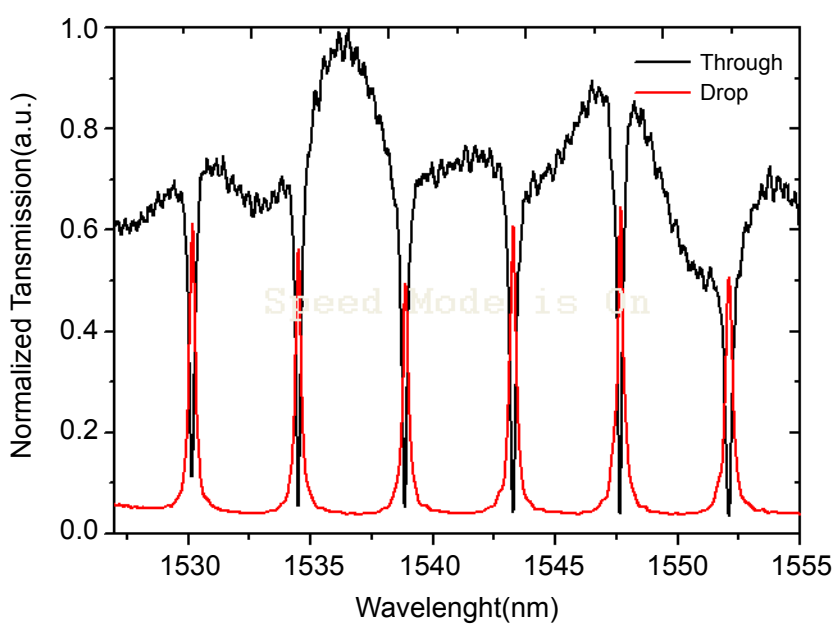

Figure 7: Normalized wavelength of two-channel sensor based on double racetrack rings.

The normalized measured responses of the double racetrack rings temperature sensor shown as Figure 7. It shows three peak responses at the length from $1529 \mathrm{~nm}$ to $1554 \mathrm{~nm}$ and the free spectral range (FSR) reaching $\sim 4.4 \mathrm{~nm}$.

\section{Conclusion}

In summary, a substantially miniaturized multi-ring photonic temperature sensor incorporating a silicon resonator was presented. A standard MEMS process was used to create the device. The influence of temperature upon the sensitivity was experimentally and theoretically examined. This device has $450 \times 220 \mathrm{~nm}^{2}$, large FSR $(18 \mathrm{~nm})$, and good linear thermo-optic effect $\left(130 \mathrm{~nm} /{ }^{\circ} \mathrm{C}\right)$. It is worth mentioning that the thermo-optic tuning bandwidth reaches $5.2 \mathrm{~nm}$ when temperature varies from 20 to $60.0^{\circ} \mathrm{C}$. And the temperature-sensitive characteristics can be used for distributed temperature sensors, filter sensors, high-speed modulators, precision optical waveguide sensors. The demonstrated sensor affords to offer salient benefits like a lowcost, homogeneous integration into other electrical/optical devices in silicon.

\section{Acknowledgements}

This work was supported by the National Natural Science Foundation of China (Grant No.61675185), the National Natural Science Foundation of Shanxi Province (Grant No.201601D011008), the Fund Program for the Scientific Activities of Selected Returned Overseas Professionals in Shanxi Province, the Program for the Top Youngand Middle-aged Innovative Talents of Higher Learning Institutions of Shanxi, and the North University of China Science Fund for Distinguished Young Scholars.

\section{References}

1. Dong P, Feng NN, Feng D, Qian W, Liang H, et al. (2010) GHz-bandwidth optical filters based on high-order silicon ring resonators. Opt Express 8: 23784-23789.

2. Irace A, Breglio G (2003) All-silicon optical temperature sensor based on MultiMode Interference. Opt Express 11: 2807-2812.

3. Shuai L, Yuanda W, Xiaojie Y, Junming A, Jianguang L, et al. (2011) Tunable filters based on an SOI nano-wire waveguide micro ring resonator. J Semicond 32: $1-5$

4. Padmaraju K, Chan J, Chen L, Lipson M, Bergman K (2012) Thermal stabilization of a microring modulator using feedback control. Opt Express 20: 27999-28008.

5. Furukawa H, Shinada S, Miyazawa T, Harai H, Kawasakiet W, et al. (2012) A multi-ring optical packet and circuit integrated network with optical buffering. Opt Express 20: 28764-28771.

6. Maru K, Abe Y (2007) Low-loss, flat-passband and athermal arrayed-waveguide grating multi/demultiplexer. Opt Express 15: 8351-18356.

7. Jin L, Zhang W, Zhang H, Liu B, Zhao J, et al. (2006) An embedded FBG sensor for simultaneous measurement of stress and temperature. IEEE Photon Technol Lett 18: 154-156.

8. Kersey AD, Berkoff TA (1992) Fiber-optic Bragg-grating differential-temperature sensor. IEEE Photon Technol Lett 4: 1183-1185.

9. Rabiei P (2003) Electro-optic and thermo-optic polymer micro-ring resonators and their applications [D]. Ph.D. Thesis, University of Southern California.

10. Dumon $P$ (2007) Ultra-compact integrated optical filters in silicon-on-insulator by means of wafer-scale technology Ph.D Thesis, University of Ghent.

11. Kim GD, Lee HS, Park CH, Lee SS, Lim BT, et al. (2010) Silicon photonic temperature sensor employing a ring resonator manufactured using a standard CMOS process. Opt Express 18: 22215-22221.

12. Teng J, Dumon P, Bogaerts W, Zhang H, Jianet X, et al. (2009) Athermal Silicon-on-insulator ring resonators by overlaying a polymer cladding on narrowed waveguides. Opt Express 17: 14627-14633.

13. Okada $Y$, Tokumaru $Y(1984)$ precise determination of lattice parameter and thermal expansion coefficient of silicon between 300 and $1500 \mathrm{~K}$. J Appl Phys 56: 314-320. 